\title{
Specification of Parameters Relevant for Trust Evaluation in an Adhoc Grid Environment
}

\author{
Slavomír Kavecký \\ Department of Informatics \\ Faculty of Management Science and Informatics \\ University of Žilina \\ Slovakia
}

\author{
Penka Martincová \\ Department of Informatics \\ Faculty of Management Science and Informatics \\ University of Žilina \\ Slovakia
}

\begin{abstract}
The purpose of security in ad hoc grid environments is to support secure execution of tasks on shared resources and to protect the resources from malicious user actions. The mechanisms of authentication and authorization commonly used in traditional grid environments are not sufficient to cover all security requirements arising from the decentralized nature of the ad hoc grid. However, the concept of trust management is capable to solve the security issues by incorporating trust into the process of decision making whether or not to execute a user's task on a selected resource. The quality of made decisions is dependent on a correct assessment and representation of trustworthiness assigned to the potentially collaborating parties. In most cases the value of trustworthiness is derived at least from direct trust and recommendations, but other factors as risk, uncertainty, context dependant information and attributes characterizing the task and the shared resource should be included in the derived value as well. This paper presents an overview of the trust evaluation process and provides a specification of parameters relevant for an accurate trust evaluation.
\end{abstract}

\section{General Terms}

Ad hoc grid computing, grid entity trust evaluation

\section{Keywords}

Grid entity trust evaluation, trust value parameters, parameters classification

\section{INTRODUCTION}

Traditional grids are based on centralized architecture, in which functional aspects as maintenance of resources, monitoring and access control enforcement are performed by a dedicated administrative authority. However, there also exists the need for support of sporadic and ad hoc communities and collaborations with dynamically changing members and access policies [1]. The motivation for ad hoc grid development is its ability to handle short-term collaborations. If a group of individuals needs to pool resources and execute computation tasks in a one-time collaboration, then administrative overhead resulting from establishment of the traditional grid environment is not practical for such a transient community. In this scenario no individual can be entrusted with administrative privileges, but still all shared resources and provided services must be protected.

Generally, the grid security protects shared resources against malicious actions of users and other entities that could damage the resources or corrupt the integrity of data stored and processed on the resources. However, in many situations the users have to be protected from those who offer the resources, so the issue is also vice-versa [5]. Grid security infrastructures incorporating only the mechanisms of authentication and authorization (often referred to as hard security mechanisms) are unable to provide this type of protection. Furthermore, the collaborations in open environments are necessarily coupled with potential dangers that necessitate reasoning about risk and uncertainty. Authentication and authorization do not allow any occurrence of risk or uncertainty in a distributed collaboration (the user is either authenticated and authorized to access a shared resource or not)

Trust is used as a mechanism for managing the potential dangers and learning from past interactions in order to reduce the risk exposure. Trust is also recognized as an important aspect of decision making in many distributed systems. For example, trust and reputation systems support decision making on the Internet provided services, which are based on a trust derived from ratings assigned to a certain provider by customers after completion of a transaction. Other parties can use the trust and reputation derived from the aggregated ratings to decide whether or not to run a transaction with the rated party in the future. Trust management (often referred to as soft security mechanism) represents the shift from attempting to provide absolute protection against potential dangers to accepting dangers as an intrinsic part of any global computing [5, 4].

The aim of the paper is to present a brief description of trust management and trust evaluation, as well as a detailed classification and specification of parameters that can be used for an accurate evaluation of trustworthiness assigned to a grid entity. The reminder of the paper is organized as follows: Section 2 provides a brief introduction to the trust management and trust evaluation; Section 3 provides classification of parameters needed for trust evaluation, describes relations between the parameters and proposes a procedure inferring the parameters into a final trust value; Section 4 describes the future work in the field; and finally, section 5 concludes the paper. 


\section{TRUST AND TRUST EVALUATION}

The notion of trust is used with variety of meanings and without any unified definition. However, in the literature are used two common definitions with well understood distinction between them. The reliability trust $[5,6]$ is defined as follows: Trust is a subjective probability by which an individual, $A$, expects that another individual, $B$, performs a given action on which its welfare depends. The decision trust $[5,6]$ is defined as follows: Trust is an extent to which one party is willing to depend on something or somebody in a given situation with a feeling of relative security, even though negative consequences are possible.

The reliability trust enables to make decisions whether or not to start a collaboration only on the basis of the collaborator's reliability estimation. On the other hand, the decision trust defines context as a part of the trust value and binds the estimation of the collaborator's reliability with a risk that arises from the uncertain outcome of the collaboration. Therefore, the decision trust seems to be a better choice for the purpose of trust modelling.

Trust between two entities is a bidirectional relationship and can be seen from multiple points of view. Humans tend to collaborate only with trusted individuals. Generally speaking, the success and survival of an entity is dependent on the willingness of other entities to collaborate. There are many genetically determined or culturally acquired strategies helping the people to appear reliable and trustworthy. The easiest and probably most used strategy for gaining trust is simply to behave in a trustworthy and reliable manner. However, the attempt to give a false impression of trustworthiness for the purpose of a personal gain is not uncommon. Therefore, it is important not only to represent own trustworthiness, but also determine correctly the trustworthiness of the target entities.

Trust management [6] is defined as follows: The activity of creating systems and methods that allow relying parties to make assessments and decisions regarding the dependability of potential transactions involving risk, and that also allow the players and system administrators to increase and correctly represent the reliability of themselves and their systems.

The parties in a computer mediated communication and collaboration need methodologies enabling them to assess properly the trustworthiness of remote parties, as well as to be recognized as trustworthy by the remote parties. This need arises due to the fact that the computer networks move the collaboration participants away from a direct style of interaction. They can collaborate with people they have never met and that they might never meet in person. Therefore, the traditional methods of trustworthiness assessment and representation used in a physical world can no longer be used. Simply expressed, the application of methodologies that enable such trusted collaborations in online environments can be called trust management [6].

\subsection{Trust Value Modelling}

Models concerned with evaluation of trustworthiness of entities in an open environments use two predominant approaches of trust evaluation: approach based on fuzzy logic and so-called discrete approach [2]. The discrete models evaluate trust either as a prediction of future collaboration outcome or the trust is calculated with help of a linear combination. Depending on the approach used to calculate the trust value, the trust models can be classified into the following three groups: (i) fuzzy logic based models, (ii) models based on a probability theory or statistical methods, (iii) and models based on linear combination.
Trust in the fuzzy logic based models is modelled as a subjective belief of the relying party about the trusted entity and expresses to what extent the relying party is willing to depend on that entity. Fuzzy logic models use linguistic terms rather than exactly calculated trust values to state how much believe an entity has in the collaborating entity. The relying party can describe the trusted entity as "Very trustworthy", "Trustworthy", "Untrustworthy" or "Very untrustworthy". In fact, the granularity of the expressions used for trust evaluation can vary. The expressions can either be defined directly in the model or the specification of the trust variable values can be left to the grid node's access policy.

The modelling approach in the fuzzy logic trust models is based on a fuzzy inference system. Grid node attributes and other relevant properties (e.g. direct trust and recommendation trust) are first transformed from crisp values into membership grades for linguistic terms of fuzzy sets. The membership functions are a subject of the designer's choice. The transformed values are processed by applying fuzzy rules provided by experts or extracted from numerical data. The output fuzzy set is processed through the process of defuzzification and the output fuzzy values are transformed into crisp values. The output values obtained from the fuzzy inference system enable to make decision on whether or not the relying party should start the transaction with other entity [2].

The outcome of actions executed by the collaborating parties in the grid environment is unknown in advance. In the models based on the probability theory the trust is related to the prediction of what that outcome will be and is estimated on the basis of previous observations.

The models based on a linear combination calculate the trust value as a combination of direct trust with reputation or as a combination of behaviour observed in past collaboration with system capabilities. However, the linear combination can also be used to combine direct trust, reputation and entity's system capabilities with other trust factors as risk, uncertainty, etc.

In an open environment it is not uncommon to start a collaboration with a completely unknown entity. However, the relying entity needs to make a decision whether or not to collaborate with that entity. If the entities in the community have no previous experiences with the unknown entity and recommendations are not available, the relying entity requires other means to evaluate the trustworthiness of the unknown entity. It could be reasoned that the lack of information about the entity makes the collaboration too risky to start. However, this precedent would disqualify any new entity joining the community from collaborating with entities already present in the community. Therefore, the initial trust was proposed as a means for determining the trustworthiness of an entity without a need for previous experiences and recommendations.

\subsection{Trust Value Structure}

Trust between two entities is formed on the basis of direct interactions between the entities and recommendations resulting from the interactions the two entities had with other entities in the grid community. Generally, each event influencing the degree of the trust is interpreted by a trusting entity as either a positive or a negative experience. If the event is interpreted as a negative experience, the trustworthiness of the trusted entity is lowered and if the event is interpreted as positive, the trustworthiness is increased by some degree[11]. The current state of the system also influences the degree of trust of the trusting entity. Therefore, the direct experiences, available recommendations and the state of the system along with risk and uncertainty are considered as factors determining the over- 


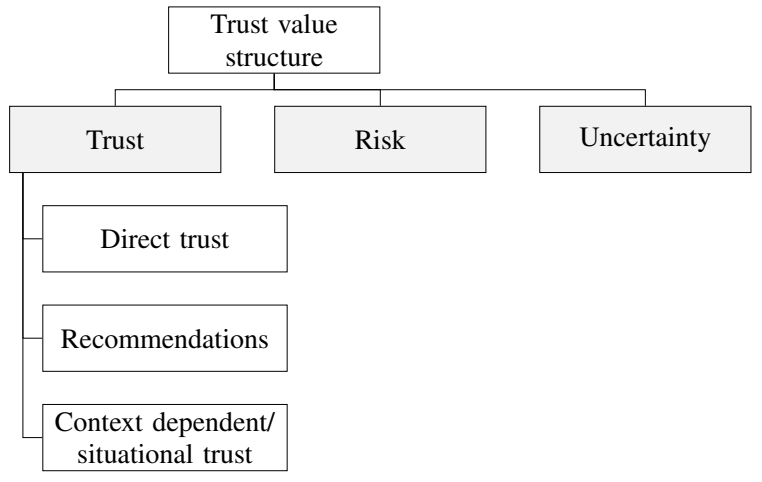

Fig. 1. Structure of the trust value and various trust relationship types among grid entities

all degree of trust and specify the various trust relationships among grid entities as depicted in Fig. 1.

The direct trust between two entities is mainly formed as a result of their previous interactions and is a part of the overall trust value in the majority of the trust models developed so far.

The recommendation trust is referred to as a reputation of the trusted entity and can be described as everything that is generally said or believed about the entity's character or standing. If the trusting entity is aware of the trusted entity's reputation it can base its trust on that reputation, i.e. the trusted entity is trusted because of its good reputation. On the other hand, if the trusting entity has a private knowledge about the trusted entity (e.g. through direct experience) and the private information overrules any reputation the trusted entity might have, then the trusted entity can be trusted despite its bad reputation.

In the trust models the situational trust is not yet fully recognized as a factor influencing the overall trust the relying entity has in the trusted entity. This type of trust relationship can be described with the following example[6]: Consider a person who distrusts a rope for climbing from the third floor of a house during a fire exercise. Imagine now that the same person is trapped in a real fire in the same house, and that the only escape is to climb from the third floor window with the same old rope. In a real fire, most people would trust the rope. In the example, the reliability trust during the fire exercise is equal to the reliability trust in a case of fire: the rope is old and therefore distrusted. However, the definition of decision trust implies that the context of a current situation in a system influences the overall trust as well. Therefore, in case of a fire the decision trust is high enough to try to use the rope to escape from the building.

Dangers are an inevitable part of any global computing system. Therefore, an explicit reasoning about the dangers causing a damage to the relying party is necessary during the process of decision making. The more important a flawless job execution is the more severe the damage in a case of failure becomes. The likelihood of a failure occurrence and the cost it incurs to the relying party is referred to as a risk. Risk and trust are related in the sense that there is no need for a trusting decision unless there is risk involved. Two alternative views of the relationship between trust and risk exist: risk determining level of trust and trust determining level of risk. The first view can be described as follows: in a particular situation or a particular action with a certain level of risk a principal should be trustworthy in order to be allowed to enter the situation or carry out the action, i.e. the level of risk determines the minimal level of required trustworthiness. The latter view is described as follows: in a particular situation or a particular action involving a principal with a certain level of trustworthiness the risk should be low enough in order to allow the principal to enter the situation or carry out the action, i.e. the level or trustworthiness determines the maximal level of acceptable risk[4]. The latter view seems more appropriate for the risk evaluation if the costs and the benefits of the entered situation are quantifiable.

Uncertainty refers to a situation where the relying party cannot be fully certain about the accuracy of the decision. In other words, the decision about possible future collaboration is done in a situation where the complete information is not present. For example, a situation can occur where two completely unknown entities have to collaborate, but they have neither the experiences with each other, nor recommendations from other entities are available. A similar situation can also occur if only a part of the information is available and other decision factors are missing. The lack of information must not necessarily result in a change of trust in the trusted entity. However, it can change the certainty about the final decision. Therefore, if the certainty is changed significantly, the level of trust is changed as well[4].

\section{PARAMETERS SPECIFICATION}

The collaborations in the grid environment are executed by two types of entities: user and resource provider. User and resource provider require protection against malicious behaviour that can take form of user's program containing malicious code capable to compromise the resource provider's node or it can take a form of a malicious resource node capable to harm the user's job running on the provided resource [8]

The security infrastructure incorporating trust should be based on a trust model that is capable to support or enhance the functional aspects of the grid infrastructure. The model should be also capable to process evidence of the previous collaborations and to transform it together with other relevant parameters into a trust value that is part of the security decisions for both the user and the resource provider protection.

\subsection{Parameters Classification}

The user and the resource provider have different requirements for the grid security infrastructure. The user is interested in competence of the shared resources to reliably execute the user's code and to protect his data from unauthorized access or modification. Similarly, the resource provider wants to collaborate only with reliable and authenticated users not compromising the shared resource or the integrity of unauthorized data.

Each participant of a collaboration in the grid environment has his own set of expectations for the quality and performance of the collaboration and is satisfied with the executed collaboration only if the required expectations were met. Trust in this context can be used to express the confidence of the relying entity that a collaborating party will meet the desired expectations. The expectations for the quality of collaboration placed by the users and resource providers are mapped to system parameters and capabilities that can be abstracted into three groups of trust components (as depicted in Fig.2): (i) behavioural parameters, (ii) system attributes (iii) and descriptive attributes.

Behavioural parameters (e.g. accessibility, availability, competence and reliability) describe the behaviour of collaborating entities and are used to create history of data obtained from past interactions. By analysing the history of the collected data using statistical methods together with the entity's personalized notion of normal or 


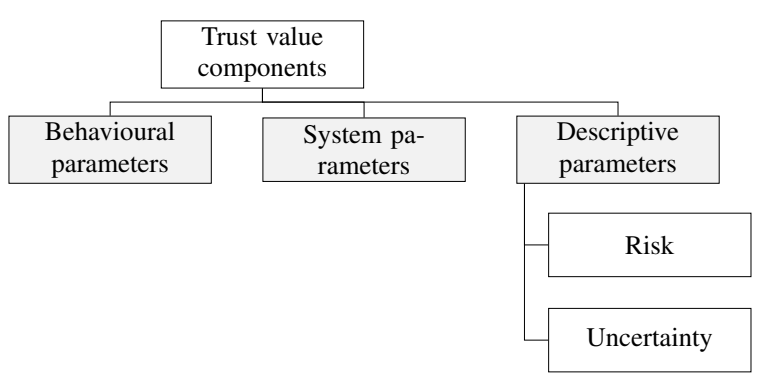

Fig. 2. Trust value components

anomalous behaviour it is possible to predict the outcome of future collaborations.

System parameters (e.g. authentication and authorization mechanism, utilized security mechanisms, maintenance of data integrity, etc.) describe the technical parameters and capabilities of the provider's shared resources and the user's node serving as access point into the grid community. The system attributes are characterized by a slow change over time. Over a period of time the attribute values do not change gradually, but the change is made suddenly and is noticeably large.

In contrast with behavioural and system attributes the descriptive parameters (e.g. benefit and loss associated with a particular collaboration, amount of observed behavioural parameters, time passed since last collaboration, etc.) do not describe the trusting disposition of the relying entity in the collaborating party, but they indicate the level of security assurance required by the relying entity. In a particular collaboration context the security assurance corresponds to the minimal trustworthiness of the collaborating party required by the relying entity.

\subsection{Determining Trust From Parameters}

As already stated, the incorporation of trust management into the grid infrastructure should support the fundamental functional aspects of the grid as resource allocation and execution of tasks. The scheduling of tasks is responsible for finding an appropriate resource node meeting the required security assurance expressed as a security demand. Similarly, the resource node declares its own security demand that must be fulfilled by the user in order to process his request by the resource node.

The security demand is dependant on the risk and uncertainty (as depicted in Fig.3) perceived by the relying party in the context of a particular collaboration. In a risky situation the relying party requires a high level of security assurance provided by the collaborating party in order to start a collaboration. Of course, the required security assurance is lower in case of a less risky situation. The uncertainty influences the security assurance in a similar manner. The higher the level of uncertainty is the less certain about a collaboration execution the relying party becomes. Therefore, the required level of security assurance increases as well.

The more important a flawless collaboration is the more severe the damage in case of failure becomes. The likelihood of failure occurrence and the cost it incurs to the relying party is referred to as a risk. Risk and trust are related in the sense that there is no need for a trusting decision unless there is a risk involved. There exist two alternative relations between trust and risk: risk determining level of trust and trust determining level of risk. The first relation can be described as follows: in a particular situation or a particular action with a certain level of risk a principal should be trustworthy in order to be allowed to enter the situation or carry out the action, i.e. the level of risk determines the minimal level of required trustworthiness. The latter relation is described as follows: in a particular situation or a particular action involving a principal with a certain level of trustworthiness the risk should be low enough in order to allow the principal to enter the situation or carry out the action, i.e. the level or trustworthiness determines the maximal level of acceptable risk. The letter view seems more appropriate for the risk evaluation if the costs and benefits of the entered situation are quantifiable [4]. The measurable parameters used for inferring the value of risk as depicted in Fig.3 are [6, 4, 7]:

Cost of a collaboration represents the price (e.g. charges for using a shared resources) a relying party has to pay, if the transaction with a collaborating party will be launched. Typically, the relying entity is not willing to invest a large amount of funds in the collaboration unless the security assurance of the collaborating party is high. Therefore, the risk perceived by the relying party increases with the growing amount of investments.

Benefit is the estimated gain (e.g. result of a data procession) obtained by the relying party after a successful execution of a collaboration. The greater the profit the more the relying party is motivated to launch a collaboration. Therefore, the risk perceived by the relying party decreases with the growing profit estimation.

Loss describes the amount of funds the relying entity will lose in case of failure. The loss is not equal only to the paid cost, but also includes the estimated benefit, lost investment of time, importance of information or data obtained through collaboration, etc. Typically, the relying party is not willing to launch a collaboration coupled with a high loss unless the security assurance of the collaborating party is high. Therefore, the risk perceived by the relying party increases with the growing loss rate.

Necessity of a collaboration execution describes a situation, in which the relying party needs to launch a collaboration in order to avoid certain or highly probably losses, even though negative consequences are possible. A high necessity of a collaboration lowers the required level of security assurance provided by the collaborating party. Hence, the risk perceived by the relying party decreases with the growing rate of a collaboration necessity.

Uncertainty refers to a situation where the relying party cannot be fully sure about the accuracy of the decision. For example, a situation can occur where two completely unknown entities have to collaborate, but they have neither the experiences with each other, nor recommendations from other entities are available. A similar situation can also occur if only a part of the information is available and other decision factors are missing. The lack of information must not necessarily result in a change of trust in the trusted entity, but it can change the certainty about the final decision. However, if the certainty is changed significantly, the level of trust is changed as well [4].

The measurable parameters used for inferring the value of uncertainty as depicted in Fig. 3 are:

Count of observations refers to the amount of data describing the behaviour of collaborating parties obtained from past interactions. With the growing rate of collaborations the knowledge about the behaviour of collaborating parties becomes more accurate. Therefore, the relying party can be more certain about the decisions based on this knowledge.

Time passed since the last collaboration determines certainty of the relaying party about its decisions. The certainty will be low, if the last interaction with a collaborating entity took 


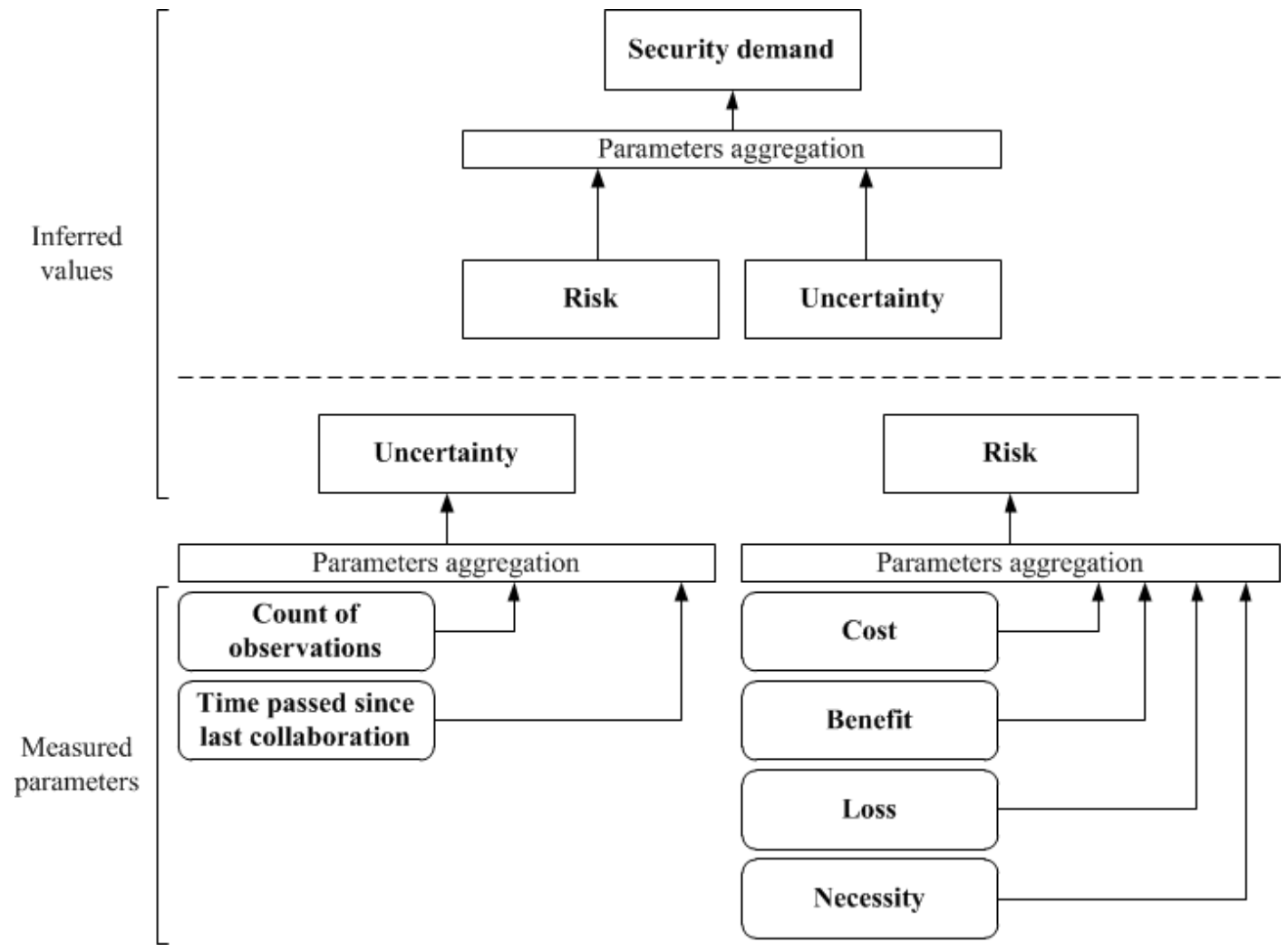

Fig. 3. Security demand inferred from trust components

place a long time ago or no collaboration was performed at all. On the other hand, if the last collaboration took place only recently, then the certainty will be high.

Trust index, which specifies the trustworthiness of a collaborating party, is dependant on direct trust and recommendations (as depicted in Fig.4). Recommendations are obtained from other entities of the grid environment and correspond to the reputation of the recommended entity. Reputation can be described as everything that is generally said or believed about the entity's character or standing. If the relying party is aware of the collaborating entity's reputation it can base its trust on that reputation, i.e. the collaborating entity is trusted because of its good reputation. Similarly, the entity becomes distrusted in case of its bad reputation.

Direct trust represents the private knowledge the relying party has about the collaborating entity and is formed from previous interactions, current context of the collaboration and attributes characterizing the collaborating entity. The direct trust and recommendations have different effect on the inferred trust index. The private knowledge of the relying entity in form of direct trust is capable to overrule the reputation of the collaborating entity, i.e. in case of high direct trust the collaborating entity is trusted despite its bad reputation and similarly, in case of low direct trust the entity is distrusted despite its good reputation. The capability of the direct trust to overrule the recommendations is dependant on the weights assigned to these two parameters.

As depicted in Fig.4, the parameters used for inferring direct trust are:

Basic trust represents the degree to which the relying party is willing to trust a collaborating entity. Before collaboration with an unknown entity the relying party assigns to that entity an initial basic trust, which characterizes the entity as half-trusted and half-untrusted. After each collaboration the value of basic trust is updated with new value that is equal to the recalculated value of trust index. Basic trust also changes over time. The more time passed since the last collaboration, the more the value of basic trust approaches its initial value.

Aggregated attributes represent the characteristics of collaborating entity relevant for trust evaluation. The attributes are aggregated into a single value corresponding to the quality of system resources offered by the entity and to the quality of entity's behaviour experienced over multiple collaborations.

Uncertainty represents the amount of available information needed by the relying party to make an accurate decision on whether or not to launch a collaboration with a particular entity. Uncertainty affects the inferred value not directly. It only determines which parameter has greater influence on the inferred value (basic trust has higher influence in case of low uncertainty and aggregated attributes affect the inferred value more in case of high uncertainty).

The aggregated attributes (as depicted in Fig.4) are inferred from system and behavioural attributes. Uncertainty represents a weighting factor determining the relative importance of the considered attributes. The impact of system attributes is higher than the impact of behavioural attributes in case of high uncertainty and the behavioural attributes affect the inferred value more significantly in case of low uncertainty.

Concrete value of aggregated attributes can be inferred from the following system attributes (as depicted in Fig.4): 


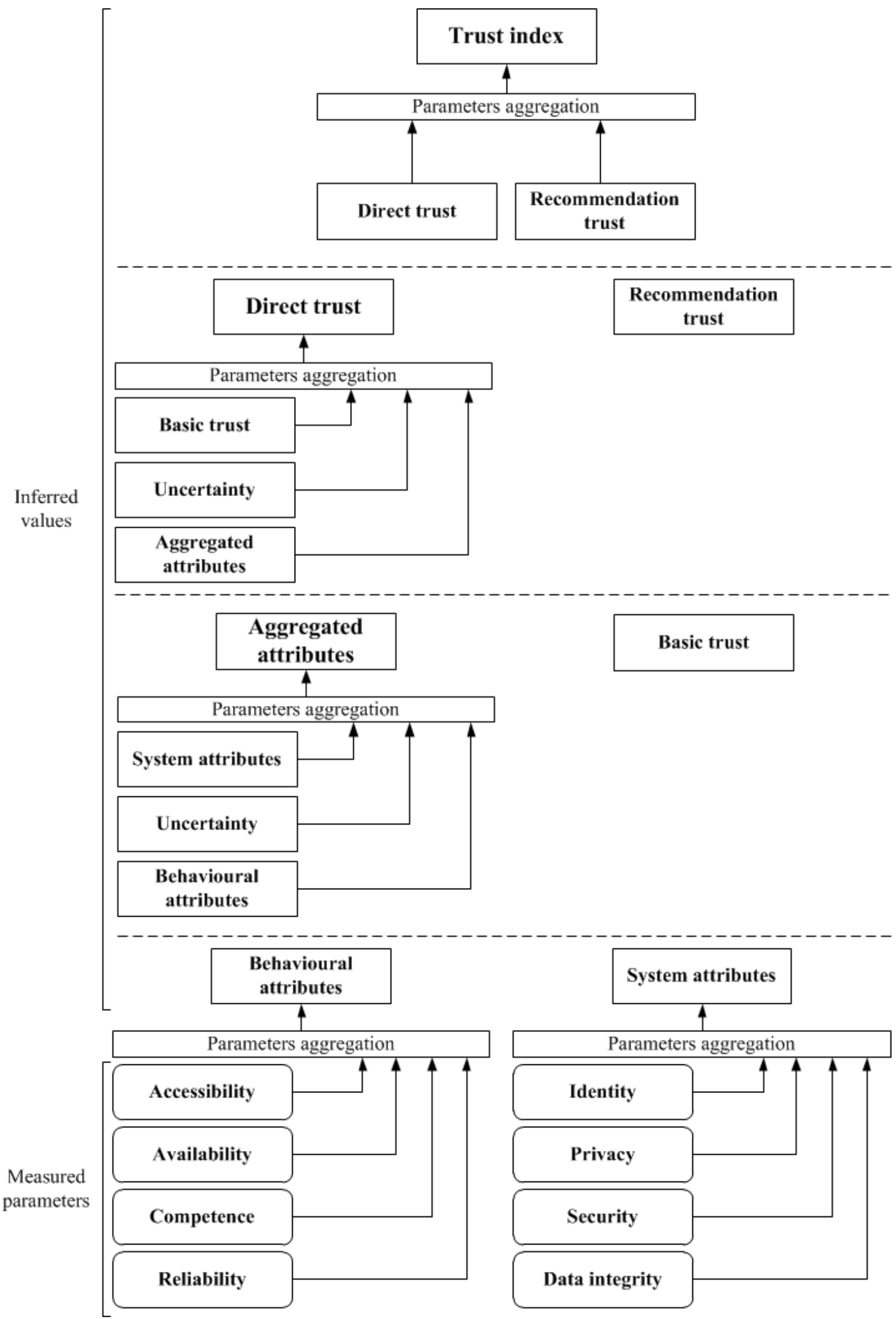

Fig. 4. Trust index inferred from trust components 
Identity describes the quality of mechanisms used by grid entities to authenticate grid users and resource providers involved in collaborations mediated through the grid environment.

Privacy corresponds to the capability of resource entity to allow access only to those system resources and data, which the authenticated user is permitted to use.

Security corresponds to the general ability of resource entity to protect itself from harm that can be caused by jobs executing malicious code, malware programs or exchange of data over communication networks.

Data integrity describes the ability of user's node and resource entity to prevent alteration of exchanged messages and data that can be avoided by protection of communication networks and by encryption of exchanged messages.

According the models dealing with behaviour trust $[10,9,3]$ the behaviour of entity can be described with the following attributes (as depicted in Fig.4):

Accessibility describes the capability of resource entity to respond to user's request for information retrieval about the state of the resource, the state of running user's job or for provision of other offered services.

Availability corresponds to the readiness of the resource to execute user's job, store data or provide other services offered by the resource provider.

Competence from user's point of view corresponds to the readiness and willingness of resource node to provide all agreed system resources specified by the user and are part of the agreement between the user and the resource provider. From provider's point of view the competence describes the user's willingness to use only the agreed system resources and to use the provided resources only for the agreed amount of time.

Reliability from user's point of view corresponds to the correct functioning of provided resource node or other services offered by the provider over a period of time. From provider's point of view the reliability describes proper execution of the user's program without compromising the provided resource node or altering unauthorized data.

\subsection{Procedure of Parameters Measurement}

Security demand and trust index, which are used for decisions making whether or not to start a collaboration, are values inferred from various system attributes, parameters describing the behaviour of collaboration participant and context dependant parameters. As depicted in the figures 3 and 4, only the parameters used for inferring behavioural attributes, system attributes, risk and uncertainty are obtained as the result of measurement. All other parameter values are inferred from parameters placed one level below (except recommendations and basic trust, which values are obtained in a separate manner).

Each of the measurable parameters are measured directly or can be broken up in measurable elements. The parameters used for inferring the value describing the behaviour of grid entity are measured directly according the entity's behaviour elements observed over multiple collaborations. Considering the collaboration participant $X$ as trustor and $Y$ as trustee, the calculated value $V_{X}$ is based on the entirety of $N$ behaviour elements under observation and is expressed according to formula 1:

$$
V_{X}=\frac{\sum_{i=1}^{N} E(Y)_{i}}{N}
$$

where $E(Y)_{i}$ represents value assigned to the $i$-th behaviour element under observation. Each observed element is calculated as the number of "positive" observations (the good behaviour was observed) divided by the total number of observations, as generalized in the formula 2 :

$$
E(Y)_{i}=\frac{\text { "positive" observations of the } i \text {-th }}{\text { element }}
$$

The parameters used for inferring the value describing the system properties of grid entity either are evaluated directly, or they are broken up in measurable and evaluable elements $[9,3]$. The identity parameter is measured according to the mechanism used for authentication of grid entities (simple password authentication, X.509 certificate authentication or Kerberos based authentication). The more sophisticated the utilized authentication infrastructure is, the higher value is assigned to the measurable element (e.g. 1, 2 or 3 respectively for the above listed authentication infrastructures). The privacy parameter is measured according to the mechanism used for authorizing users to access only allowed resources and data (simple password authorization, identity based authorization or role based authorization). The more advanced the used authorization infrastructure is, the higher value is assigned to the measurable element (e.g. 1, 2 or 3 respectively for the above listed authorization types).

The security parameter is calculated as aggregation of the following measurable elements: self protection, secure network connection and provision of execution sandbox. The self protection of grid entity is measured according to mechanisms utilized by the entity (virus and malware protection, firewall and intrusion detection system) to protect itself from malicious activities of collaborating parties. The more mechanisms the entity employs, the higher the value of self protection is (e.g. the element value is increased by one for each utilized mechanism). The secure network connection is measured according to the type of protocol suite utilized for securing the communication between collaborating entities (TLS or IPSec). The more advanced the used protocol suite is, the higher value is assigned to the measurable element (e.g. 1 or 2 respectively for the above listed protocol types). The provision of execution sandbox is evaluated according to the capability of resource entity to run user programs in a tightly controlled environment (e.g the measurable element is assigned 1 if the sandbox is provided, otherwise the element is assigned 0 ).

The data integrity parameter is calculated as aggregation of the following measurable elements: data corruption protection and secure network connection. The data corruption protection is measured according the mechanisms used to prevent unintended changes in data that can occur during writing, storing, reading, or processing (hardware RAID solution or internal data and metadata checksumming). The more mechanisms the entity employs, the higher the value of data corruption protection is (the element value is increased by one for each utilized mechanism). The secure network connection is measured in the same way as the secure network connection in case of security parameter (i.e according the type of utilized protocol suite).

Considering the collaboration participant $X$ as trustor and $Y$ as trustee, the value of inferred system attributes $V_{X}$ is based on the entirety of $N$ measured system parameters and is expressed according to formula 1 where $E(Y)_{i}$ represents value assigned to the $i$-th evaluated system parameter. The value of identity and privacy parameters is calculated as the value assigned to measurable element associated with the system parameter divided by the maximal at- 
tainable value as generalized in formula $3:$

$$
E(Y)_{i}=\frac{\begin{array}{c}
\text { value assigned to the } i \text { - } t h \\
\text { measurable element }
\end{array}}{\begin{array}{c}
\text { maximal attainable value } \\
\text { of the } i \text {-th element }
\end{array}}
$$

In case of security and data integrity parameters, the value $E(Y)_{i}$ is calculated as the sum of $n$ values assigned to the measurable element associated with the system parameter divided by the maximal value attainable by the measurable element as generalized in formula 4:

$$
E(Y)_{i}=\frac{\sum_{j=1}^{n} \begin{array}{c}
j \text {-th value assigned to the } i \text {-th } \\
\text { measurable element }
\end{array}}{\begin{array}{c}
\text { maximal attainable value } \\
\text { of the } i \text {-th element }
\end{array}}
$$

The parameters used for inferring the value of uncertainty are measured straightforwardly as explained in section 3.2. Only the values of parameters used for inferring the risk are obtained in a different manner. The user and the resource provider define their collaboration dependant expectations that are reflected in the risk parameters (e.g. what is the expected cost of executed collaboration, what benefits can be achieved by the collaboration, how important is the execution of collaboration for the user or the resource provider, or how much damage the user or the resource provider will take in case of collaboration failure).

The attribute values inferred from the measurable parameters are normalized to fit into the interval $[0,1]$. The reason for the value normalization is the difference in the maximal values assignable to the inferred attributes. After the normalization the attributes can be combined either with linear combination or with combination based on fuzzy logic. The concrete weighting factors used in linear combination or concrete rules used in fuzzy logic can be modified to reflect the expectations of user and resource provider and the context of future collaboration. However, the weighting factors and fuzzy rules should still take into account the relations among the attributes described in the section 3.2.

\section{FUTURE WORK}

As stated in the article, the requirements for the grid security can be mapped to various system, behavioural and descriptive attributes specifying the capability of a collaborating entity to execute activities in a secure manner. However, the participants of the grid community usually have different requirements for the grid security. The diversity of the requirements necessitates the ability of the collaborating participants to define what system and/or behavioural attributes of interest should be included in the evaluated trust value. Similarly, the participants should be also able to assign weights to the evaluated attributes in order to specify the desired impact of the attributes on the evaluated trust value.

The task for the future research is to propose an infrastructure giving the grid community participants an ability to specify the evaluated attributes and their weights in an easy and understandable manner (e.g. this issue is addressed by Dionysiou and Gjermundrod [3]). The infrastructure should also provide an well-defined application interface enabling an easy integration of trust management (i.e. trust evaluation, trust update and decisions based on trust) into the current ad hoc grid solutions.

\section{CONCLUSION}

The procedure of trust evaluation is a complex process including procession of various trust components and relations among these components. The paper describes in detail these relations and their impact on the inferred values produced by the trust management inference system. The values are deduced from parameters describing the most significant system attributes and behavioural traits of evaluated grid entity (namely behavioural parameters, system parameters, risk and uncertainty). The paper also provides an overview of various system attributes, behavioural and descriptive parameters that are either measured directly or can be broken up in measurable elements. Description of general procedure used for measuring and inferring the trust components is introduced as well.

\section{REFERENCES}

[1] Kaizar Amin, Gregor von Laszewski, and Armin R. Mikler. Toward an architecture for ad hoc grids. In 12th International Conference on Advanced Computing and Communications (ADCOM 2004), Ahmedabad, pages 15-18, 2004.

[2] Michael Brinklov and Robin Sharp. Incremental trust in grid computing. In Proceedings of the Seventh IEEE International Symposium on Cluster Computing and the Grid, CCGRID '07, pages 135-144, 2007.

[3] Ioanna Dionysiou and Harald Gjermundrod. sguts: Simplified grid user trust service for site selection. In The Seventh International Conference on Internet Monitoring and Protection, 2012, pages 40-46, May 2012.

[4] Colin English, Sotirios Terzis, and Waleed Wagealla. Engineering trust based collaborations in a global computing environment. In Trust Management, volume 2995 of Lecture Notes in Computer Science, pages 120-134, 2004.

[5] Audun Jøsang, Roslan Ismail, and Colin Boyd. A survey of trust and reputation systems for online service provision. Decision Support Systems, 43(2):618-644, mar 2007.

[6] Audun Jøsang, Claudia Keser, and Theo Dimitrakos. Can we manage trust? In Trust Management, volume 3477 of Lecture Notes in Computer Science, pages 93-107. Springer Berlin Heidelberg, 2005.

[7] Audun Jøsang and Stéphane Lo Presti. Analysing the relationship between risk and trust. In Trust Management, volume 2995 of Lecture Notes in Computer Science, pages 135-145. Springer Berlin Heidelberg, 2004.

[8] Ching Lin, Vijay Varadharajan, Yan Wang, and Vineet Pruthi. Enhancing grid security with trust management. In Proceedings of the 2004 IEEE International Conference on Services Computing, 2004., pages 303-310, Sept 2004.

[9] P.D. Manuel, S. Thamarai Selvi, and M.IA-E. Barr. Trust management system for grid and cloud resources. In First International Conference onAdvanced Computing, 2009., pages 176-181, Dec 2009.

[10] Elvis Papalilo and Bernd Freisleben. Managing behaviour trust in grid computing environments. Journal of Information Assurance and Security, 3:27-37, March 2008.

[11] Jianqiang Shi, Gregor v. Bochmann, and Carlisle Adams. A trust model with statistical foundation. In Formal Aspects in Security and Trust, volume 173 of IFIP International Federation for Information Processing, pages 145-158. Springer US, 2005. 